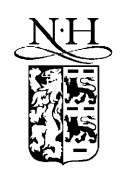

\title{
QCD glueball Regge trajectory and the pomeron
}

\author{
Felipe J. Llanes-Estrada ${ }^{\text {a }}$, Stephen R. Cotanch ${ }^{\text {a }}$, \\ Pedro J. de A. Bicudo ${ }^{\text {b }}$, J. Emilio F.T. Ribeiro ${ }^{\text {, }}$, \\ Adam Szczepaniak $^{\mathrm{c}}$ \\ a Department of Physics, North Carolina State University, Raleigh, NC 27695-8202, USA \\ ${ }^{\mathrm{b}}$ Departamento de Física and Centro de Física das Interações fundamentais, Instituto Superior Técnico, \\ Av. Rovisco Pais, 1049-001 Lisboa, Portugal \\ ${ }^{\mathrm{c}}$ Department of Physics and Nuclear Theory Center, Indiana University, Bloomington, IN 47405, USA
}

Received 13 February 2002; received in revised form 27 June 2002; accepted 17 July 2002

\begin{abstract}
Implementing many-body techniques successful in other fields, we report a glueball Regge trajectory emerging from diagonalizing a confining Coulomb gauge Hamiltonian for constituent gluons. Through a BCS vacuum ansatz and gap equation, the dressed gluons acquire a dynamic mass, of order $0.8 \mathrm{GeV}$, providing the quasiparticle degrees of freedom for a TDA glueball formulation. The TDA eigenstates for two constituent gluons have orbital, $L$, excitations with a characteristic energy of $0.4 \mathrm{GeV}$ revealing a clear Regge trajectory. In particular, the $J^{P C}=2^{++}$glueball coincides with the pomeron given by $\alpha_{P}(t)=1.08+\left(0.25 \mathrm{GeV}^{-2}\right) t$. We also ascertain that lattice data supports our result. Finally, we conjecture on the odderon puzzle.

(c) 2002 Elsevier Science B.V. All rights reserved.
\end{abstract}

PACS: 11.55.Jy; 12.39.Mk; 12.39.Pn; 12.40.Yx

Keywords: Glueball Regge trajectories; Pomeron; Odderon; QCD; Coulomb gauge Hamiltonian; TDA

\section{Introduction}

A vintage problem in hadronic physics is to fundamentally explain the observed monotonously rising, to asymptotically flat cross section behavior with increasing Mandelstam variable $s$. Pre-QCD cross section theorems [1] involving $t$ channel exchanges motivated Pomeranchuk's assertion [2] that vacuum quantum number $(P C=++) t$

E-mail address: cotanch@ncsu.edu (S.R. Cotanch). 
channel exchanges would describe elastic cross section high energy dependence. Related, it was historically known that hadronic resonances with specific quantum numbers could be connected to a series or "tower" of excited states. From this backdrop the successful Regge picture [3,4], with poles and trajectories linear in $t$ (mass squared), $\alpha(t)=\alpha(0)+b t$, emerged which effectively described cross sections and resonances and therefore unified both scattering and bound state data. Of particular interest in this paper are analyses of high energy diffraction data [5-7] yielding a $J^{P C}=J^{++}$Regge trajectory with the largest intercept $\alpha(0)$. This trajectory is called the pomeron and from detailed fits is given by

$$
\alpha_{P}(t)=1.08+\left(0.25 \mathrm{GeV}^{-2}\right) t .
$$

Note that by definition, the pomeron entirely governs and correctly reproduces the high energy scattering behavior since the cross section scales as $s^{\alpha(0)-1}$. The simplicity of this picture is very appealing and the pomeron remains a contemporary tool for understanding high energy processes, both experimentally and theoretically. Here we specialize to the soft, non-perturbative pomeron which dominates in the analysis of high $s$ diffractive data [8]. The hard, perturbative pomeron is relevant at a higher (hard) energy scale, such as large $Q^{2}$, large $t$ [9] or charm production. This scale is above our present study, especially high $t$ which is clearly beyond the domain of our glueball formulation presented below (for an account of the BFKL hard pomeron in perturbative QCD see [10-12]).

According to Regge theory, the trajectory describing scattering should also be consistent with physical hadron states, implying a $J^{P C}=J^{++}$trajectory of mesons with slope near $0.25 \mathrm{GeV}^{-2}$. However, all known hadronic resonances (both mesons and baryons) fall on Chew-Frautschi [13] trajectories with slopes close to $0.9 \mathrm{GeV}^{-2}$ but different intercepts. This is illustrated in Fig. 1 for several mesons including the $\rho$ whose negative parity, positive $G$ parity tower is well described by $\alpha_{\rho}(t)=0.55+\left(0.9 \mathrm{GeV}^{-2}\right) t$. This incompatibility led to the conjecture that the pomeron corresponded instead to a Regge trajectory for gluonic states, and most likely one describing the $J^{++}$glueballs.

Not surprisingly, the gluonic nature of the pomeron has been widely investigated (see [14] for a recent review) with several recent studies linking the pomeron and glueball trajectories. From meson trajectories and Wilson loops, Kaidalov and Simonov [15] estimate that the glueball intercept is $\alpha(0) \simeq 0.7$, while Soloviev [16] computes $\alpha(0)=$ $1.07 \pm 0.03$ using the quantized elliptic Nambu-Gotto string model. Motivated by the Maldacena conjecture, and solving the dilaton wave equation, Brower, Mathur and Tan [17] obtained a glueball intercept of $\alpha(0) \simeq 1.2$. Brisudova, Burakovsky and Goldman [18] used mass relations between the glueball and meson sectors to obtain a glueball Regge trajectory with slope $\simeq 0.3 \pm 0.1 \mathrm{GeV}^{-2}$. Finally, as noted by Donnachie and Landshoff [5], there is experimental evidence for a $2^{++}$glueball [19] with mass near $2 \mathrm{GeV}$ which is consistent with the pomeron. See also Ref. [20] for even more recent measurements supporting a $2^{++}$glueball mass of $1.95 \mathrm{GeV}$.

In this communication we report results from a new constituent glueball approach which further strengthens the gluonic pomeron conjecture. Similar to previous relativistic quark approaches [21,22] yielding a consistent meson Chew-Frautschi Regge slope, our relativistic many-body calculations also produce a glueball Regge trajectory that arises naturally from the confining linear potential and the scale of angular excitations. In reproducing the lattice gauge positive parity glueball spectrum, we obtain a Regge 


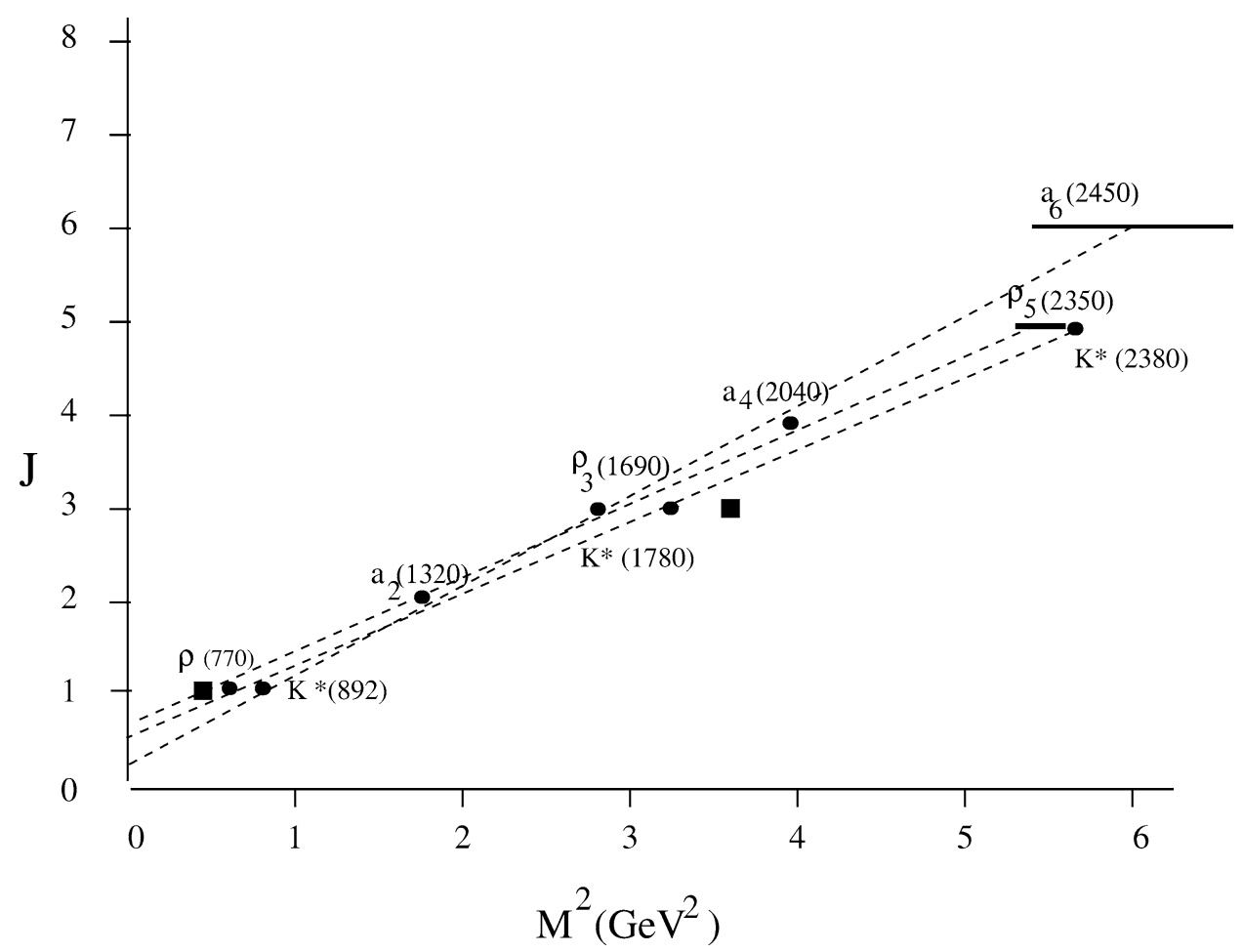

Fig. 1. Meson $\left(\rho, K^{*}\right.$ and $\left.a\right)$ Regge trajectories constructed from recent tabulated data (dark circles and error bars, PDG 2000). Boxes are model TDA predictions for the $\rho$ trajectory.

trajectory with slope close to the pomeron value $\left(b \approx 0.25 \mathrm{GeV}^{-2}\right)$ and predominantly independent of model details. In particular, our $2^{++}$glueball prediction coincides with the pomeron. Our group has applied many-body techniques to develop a unified approach for the hadron spectrum utilizing a QCD inspired Hamiltonian. Both the gluon [23] and meson $[24,25]$ sectors have been realistically described and calculations for both exotic and charmed hybrid mesons have also recently been published [26].

\section{Effective Hamiltonian, gap equation and glueball spectrum}

We briefly summarize our many-body approach (see Refs. [23-26] for complete calculational details). The starting point is a Coulomb gauge, field theoretical Hamiltonian

$$
H=\operatorname{Tr} \int d \mathbf{x}\left(\boldsymbol{\Pi}^{a} \cdot \boldsymbol{\Pi}^{a}+\mathbf{B}_{A}^{a} \cdot \mathbf{B}_{A}^{a}\right)-\frac{1}{2} \int d \mathbf{x} d \mathbf{y} \rho^{a}(\mathbf{x}) V(|\mathbf{x}-\mathbf{y}|) \rho^{a}(\mathbf{y}),
$$

with color charge density $\rho^{a}=f^{a b c} \mathbf{A}^{b} \cdot \boldsymbol{\Pi}^{c}$ and fields $\boldsymbol{\Pi}^{a}, \mathbf{B}_{A}^{a}=\nabla \times \mathbf{A}^{a}$. We utilize an instantaneous Cornell potential for confinement, $V=-\frac{\alpha_{s}}{r}+\sigma r$. The potential parameters are $\alpha_{s}=0.2$, and, from independent lattice results, $\sigma=\frac{3}{4} 0.18 \mathrm{GeV}^{2}$. The gluons are then 
dressed by means of a Bardeen, Cooper and Schrieffer (BCS) ansatz for the vacuum ground state which, through a variational Hamiltonian minimization, generates the gap equation

$$
\omega_{q}^{2}=q^{2}-\frac{3}{4} \int \frac{d \mathbf{k}}{(2 \pi)^{3}} \widehat{V}(|\mathbf{q}-\mathbf{k}|)\left(1+(\hat{\mathbf{k}} \cdot \hat{\mathbf{q}})^{2}\right)\left(\frac{w_{k}^{2}-w_{q}^{2}}{w_{k}}\right),
$$

for the gluon self-energy, $\omega_{q}$, containing the constituent gluon mass. It is important to stress that our initially massless gluons acquire mass dynamically just as current quarks are dressed [23-26]. Our potential in momentum space is $\widehat{V}(|\mathbf{q}-\mathbf{k}|)=-\frac{4 \pi \alpha_{s}}{(q-k)^{2}}-\frac{8 \pi \sigma}{(q-k)^{4}}$, where each term generates a divergence in the gap equation (Coulomb is quadratic, confinement is logarithmic). Since we are focusing on confinement we have omitted the Coulomb potential in solving Eq. (3) and then used a cut-off parameter $\Lambda=4-5 \mathrm{GeV}$ to regularize the remaining logarithmic divergence. Finally, a Tamm-Dancoff (TDA) diagonalization, truncated to the $1 \mathrm{p}-1 \mathrm{~h}$ level, is performed for the same field theoretical Hamiltonian (no divergences, Coulomb potential is now included) to generate the glueball spectrum. We used the same linear potential in our quark sector applications, which properly implement chiral symmetry, to produce reasonable results [24-26], including the appropriate meson Regge slope as indicated by the solid squares in Fig. 1 for the $1^{--}$and $3^{--} \rho$ trajectory.

At the more technical level, the glueball TDA state, for fixed orbital, $L$, and total gluon spin, $S$, is represented by

$$
\left|\Psi_{L S}^{J P C}\right\rangle=\sum_{a m_{1} m_{2}} \int \frac{d \mathbf{q}}{(2 \pi)^{3}} \Phi_{L S m_{1} m_{2}}^{J P C}(\mathbf{q}) \alpha_{m_{1}}^{a \dagger}(\mathbf{q}) \alpha_{m_{2}}^{a \dagger}(-\mathbf{q})|\Omega\rangle,
$$

where the $\alpha_{m}^{a \dagger}$ are quasiparticle creation operators acting on the BCS vacuum state $|\Omega\rangle$. The sum is over the color index $a$ and the two transverse spin projections, $m=1,2$. The glueball wavefunction has angular momentum composition

$$
\Phi_{L S m_{1} m_{2}}^{J P C}(\mathbf{q})=\left\langle 1 m_{1} 1 m_{2} \mid S m_{S}\right\rangle\left\langle L m_{L} S m_{S} \mid J m_{J}\right\rangle \phi_{L S}^{J P C}(q) Y_{L}^{m_{L}}(\hat{\mathbf{q}}) .
$$

As mentioned above, the gluon quasiparticle energy is obtained from the non-linear mass gap equation which yields a gluon mass around $0.8 \mathrm{GeV}$. Then, with the gap energy, the linear TDA equations are diagonalized for the $J^{P C}$ states of interest, either as a radial equation or variationally in a multi-dimensional Monte Carlo calculation for the Hamiltonian matrix elements using the computer code VEGAS. Using a new, independent code we have recomputed the glueball spectrum and confirmed our original results [23]. While our work omits renormalization, a recently renormalized glueball calculation [27] produced similar $J^{P C}=0^{ \pm+}$predictions. We have also extended the analysis to the maximally acceptable $1 \mathrm{p}-1 \mathrm{~h}$ excitation range spanning higher spin states, up to $3^{++}$. The higher angular momentum significantly enhances the interaction spin dependence, including spin-orbit, which is not accurately known. The $1 \mathrm{p}-1 \mathrm{~h}$ energy upper bound is about $3 \mathrm{GeV}$ since $2 \mathrm{p}-2 \mathrm{~h}$ ( 4 gluon) states can become important for energies around twice the lightest $1 \mathrm{p}-1 \mathrm{~h}$ excitation $(\approx 1.6 \mathrm{GeV}$ ground state glueball). A realistic calculation above $3 \mathrm{GeV}$ may entail mixing with $2 \mathrm{p}-2 \mathrm{~h}$ states requiring a complicated (4 gluon) Fock space diagonalization. Also potentially important will be excited $C=+$ three gluon states discussed further below. All of this will be addressed in a future work and it will be of 


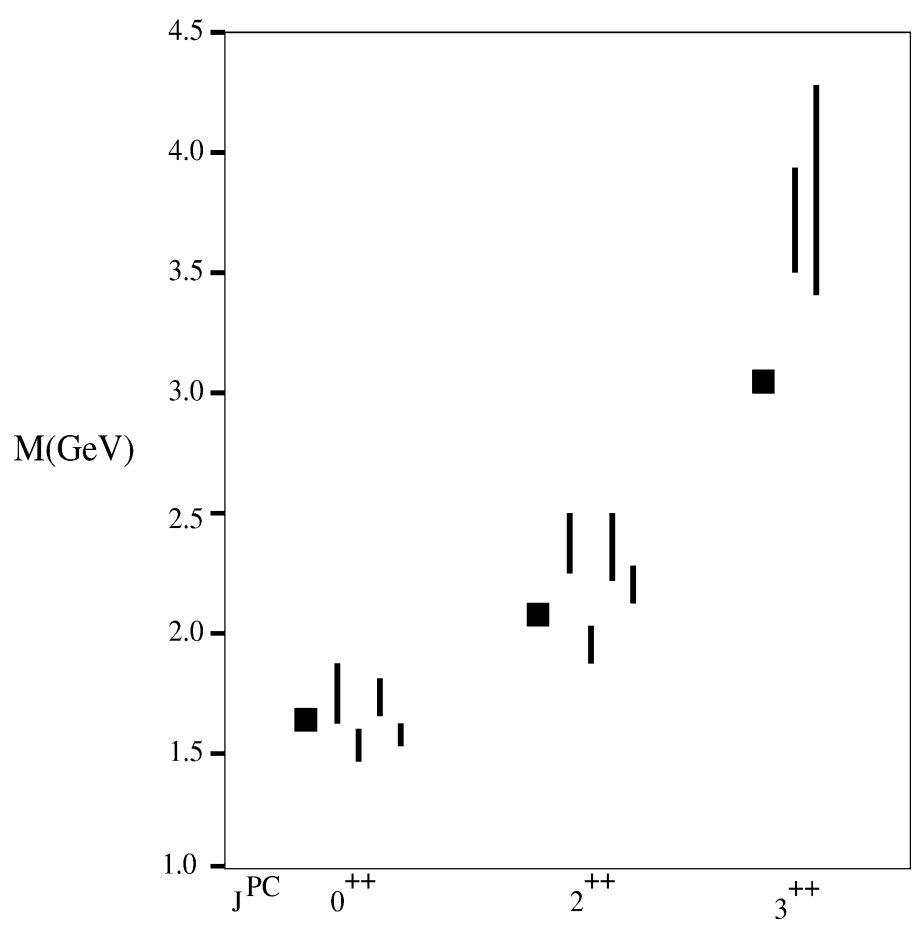

Fig. 2. The TDA (boxes) and lattice gauge (vertical bars showing error) glueball spectra. The lattice results, from left to right, are from Refs. [28-31], respectively.

interest to determine if there is an appreciable shift in the energy levels. For these reasons we have limited the scope of our meson spectrum prediction to spin $J=3$ or less as shown in Fig. 1.

Our glueball predictions are compared to previous and most recent lattice gauge measurements [28-31] in Fig. 2. To avoid confusion with Regge trajectory plots the more conventional vertical axis is used for the mass spectrum in Fig. 2 which is reversed from Fig. 1. Note that while our $0^{++}$and $2^{++}$states reliably reproduce the lattice results, our $3^{++}$state is too low which is consistent with above remarks. We comment on this state further in the next section.

\section{Glueball Regge trajectories and the pomeron connection}

With this technical prelude we now address the relation of the glueball to the pomeron. Three constituent gluon states are not considered since they are expected to be more massive than a two gluon glueball and thus on daughter trajectories (see Section 4). Related, a three gluon $P C=++$ state involves excited (non s wave) antisymmetric spacespin (with antisymmetric color) configurations which would further increase its mass. Wavefunction symmetry for two identical bosons requires that $L+S$ must be even and since the pomeron has positive parity, both $L$ and $S$ are individually even. Also, for a 


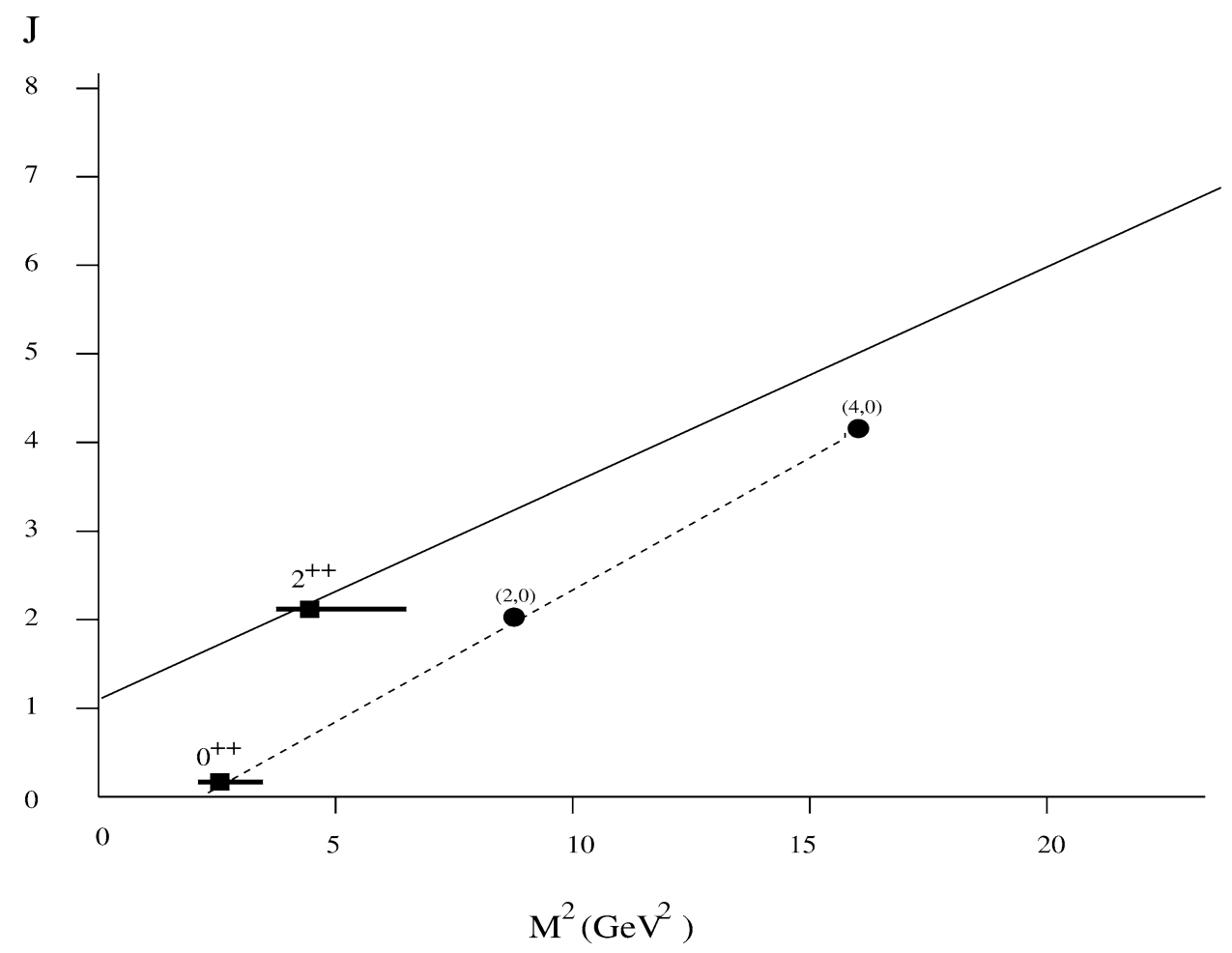

Fig. 3. Comparison of TDA (boxes) and lattice (horizontal bars) $2^{++}$and $0^{++}$glueballs with the pomeron (solid line) and daughter (dashed line) Regge trajectories, respectively. The slope of the daughter trajectory is $0.28 \mathrm{GeV}^{-2}$ and was determined by the higher $(L=2, S=0)$ and $(L=4, S=0)$ orbital excitations (solid circles).

two gluon system Yang's theorem, a combination of gluon transversality (Coulomb gauge) and Bose statistics, forbids a $J=1$ state. Further, the $J=0$ state is precluded from the pomeron since it necessarily belongs to a lower (daughter) trajectory. Lastly, the pomeron has even signature (for bosons, signature $=(-1)^{J}$ ). Therefore only the $J=2^{++}$state $(L=0)$ in Fig. 2 is a pomeron candidate and the corresponding glueball Chew-Frautschi diagram is shown in Fig. 3 along with the pomeron trajectory.

Fig. 3 constitutes the key finding of this study. Notice that our model yields a glueball reggeon (upper dark box) in reasonable agreement with the pomeron. Equally significant is the lattice $2^{++}$data which quantitatively supports our result. We therefore conclude that both our model and recent lattice data are consistent with a glueball interpretation of the pomeron.

Concerning the $3^{++}$glueball, the theoretical uncertainty in our model and lattice mass predictions makes it difficult to assign this state to a specific Regge trajectory. If there is a glueball pomeron connection then due to the $3^{++}$odd signature it cannot be on the even signature pomeron and must be on a daughter trajectory. Further, this daughter trajectory cannot be exchange degenerate with the pomeron since even and odd signature trajectories 
having the same parity (and charge conjugation) destructively contribute to the scattering process. This would yield, for example, a suppressed high energy proton-proton scattering cross section with no pomeron signature which is inconsistent with observation. This is similar to the quark sector where the odd signature $a_{1}(1260)$ and $f_{1}(1285)$ mesons are on non-degenerate daughter trajectories distinct from the even signature $a_{J}$ and $f_{J}$. See Ref. [4] for a complete discussion. Using the $3^{++}$lattice mass, between 3.3 and $4.3 \mathrm{GeV}$, yields a trajectory point (not shown) below the pomeron suggesting that this state is on a daughter trajectory as it should be. While exchange degeneracy between same parity trajectories is not expected, opposite parity trajectories can be degenerate in potential models like ours (e.g., see Fig. 1 where the $\rho$ and $a$ meson are weakly degenerate). Although lattice calculations do not indicate clear glueball reggeon candidates, if such a trajectory exists close to the pomeron it might yield a measurable signal, especially in processes involving two pomeron exchange. This may be related to the recent observation [32] of unexpected azimuthal angle dependence in $p p$ central meson production which is consistent with the hypothesis [33] that the pomeron transforms as a non-conserved vector current. Clearly, these issues require further study and we will report in a future communication results using an improved spin interaction.

Ideally, higher $\mathrm{J}^{++}$states should also be accurately predicted. While currently too formidable for our approach, a very recent lattice calculation [34] reports the value $3.65 \mathrm{GeV}$ for the $4^{++}$glueball. This is very close to the pomeron value, $M_{J}=$ $2 \sqrt{J-1.08}=3.42 \mathrm{GeV}$, for $J=4$. Using this and the other, but still uncertain, lattice $2^{++}$mass yields a lattice predicted slope of $0.23 \mathrm{GeV}^{-2}$ and intercept 0.94 . Considering the lattice error, this is in reasonable agreement with the values in Eq. (1) and further strengthens the glueball pomeron interpretation. It would indeed be worthwhile to have independent lattice confirmations of the $4^{++}$mass and also predictions for the $J=6$ mass to see if it too is near the pomeron trajectory value, $M_{J}=2 \sqrt{J-1.08}=4.44 \mathrm{GeV}$. Although experimentally challenging, observation of a $4^{++}$glueball with mass near $3.5 \mathrm{GeV}$ would also be further affirming.

Although we are not yet able to calculate an intercept from our analysis we can compute the Regge slope which is most unambiguously obtained by extending the $S=0,0^{++}$ glueball (lower dark box in Fig. 3) trajectory to the $L=2, S=0$ and $L=4, S=0$ states. Such configurations minimize uncertainties from spin dependence (no spin-spin, spinorbit or tensor contributions). The resulting daughter trajectory is represented by the dotted line in Fig. 3 and yields a slope of $0.28 \mathrm{GeV}^{-2}$. This slope is characteristic of our potential model (see discussion below) and is essentially the same for other $S \neq 0$ trajectories having different intercepts (not shown). We found limited sensitivity of our results to the potential parameters as increasing $\sigma$ and $\alpha_{s}$ by $20 \%$ to $30 \%$ only altered the trajectory by $10 \%$ to $20 \%$.

A major advantage of our model is the dynamical insight attending the unified quark/gluon prediction of meson and pomeron slope trajectories with respective values $b_{q \bar{q}} \approx 0.9 \mathrm{GeV}^{-2}$ and $b_{g g} \approx 0.25 \mathrm{GeV}^{-2}$. In a simple QCD string model with massless partons separated by a string with length $r$ and tension (energy per unit length) $k$, the total relativistic mass scales as $k r$ while the angular momentum is proportional to $\frac{1}{2} k r^{2}$, which generates a Regge trajectory with slope $b=\frac{1}{2 \pi k r}$. This string feature is incorporated in our model through the linear confining potential $(\sigma r)$, yielding effective string tensions, 
$k_{q \bar{q}}=C_{q \bar{q}} \sigma$ and $k_{g g}=C_{g g} \sigma$, for the meson and glueball systems, respectively. Here $C_{q \bar{q}}=\frac{N_{c}^{2}-1}{2 N_{c}}$ and $C_{g g}=N_{c}$ are the respective quark and gluon color Casimir operators from the density-density Hamiltonian kernel. Hence for $N_{c}=3$ the meson and glueball

trajectory slopes are related by $b_{g g}=\frac{C_{q \bar{q}}}{C_{g g}} b_{q \bar{q}}=\frac{4}{9} b_{q \bar{q}}$. While this simple estimate explains much of the meson/glueball slope difference, it is significant that our model is able to account for entirely all of the difference, the rest due to BCS quasiparticle masses and the Coulomb potential in the TDA. It would be very interesting to see if alternative QCD models, particularly the flux tube, can reproduce these results.

\section{Odderon conjecture}

Finally, we comment on the controversial odderon [35], the odd signature, $P C=--$ partner to the pomeron. If the odderon and pomeron have similar trajectories, it is puzzling why the soft odderon has not been observed at $t=0$. In contrast to the pomeron having $C=+$, odderon Regge pole exchange would produce differences between $p p$ and $\bar{p} p$ scattering at high $s$ and low $t$. However, high energy cross section measurements reveal small, if any, differences and the most recent, comprehensive high energy $t=0$ data [36] excludes a $C=-$ trajectory with intercept near unity. Assuming the odderon exists, one argument for its absence in nucleon scattering is suppression of odderon-proton coupling due to diquark formation [37].

Here we conjecture with an alternative explanation based upon our model which would represent the odderon as a three gluon system. From our previous three body studies of baryons $(q q q)$ and hybrid mesons $(q \bar{q} g)$, we suspect our model would also generate a Regge trajectory for the odderon with slope similar to the pomeron. However, even if the slope is not similar, our approach would yield an odderon intercept smaller than 1.08 since a three gluon glueball would be heavier than a two gluon state by at least the mass of a constituent gluon, of order $0.8 \mathrm{GeV}$. Lattice results [28,29] support our conjecture as the lightest measured $C=-$ glueball has mass around $3 \mathrm{GeV}$ and the $3^{--}$lattice mass is $4.13 \mathrm{GeV}$ (the $1^{--}$lattice mass is $3.85 \mathrm{GeV}$ and would appear to be on a different, daughter trajectory). A larger mass shifts the trajectory to the right, lowering the intercept, and we would estimate that our model odderon Regge trajectory would have an intercept closer to 0 (possibly even negative) instead of 1 . The odderon would therefore not be competitive with the pomeron in governing high energy behavior and, depending on the odderon intercept value, would only be observed at lower energies. A more precise analysis is in progress.

If indeed the odderon, or other $C=-1$ trajectory, which requires at least 3 gluons in our model, exists with an intercept near or above unity, our constituent gluon/glueball approach would be undermined, especially if a very light $C=-1$ glueball was observed. However, if our conjecture is correct, identification of the odderon would be difficult especially if its intercept is below 0.5 since it would now also compete with $\omega$ exchange. However, if the odderon intercept is larger than 0.5 then it may still be possible to observe it through pseudoscalar [38] and tensor [39] meson electromagnetic production where there are no pomeron contributions. Such experiments should prove interesting, especially if they are able to provide details on the odderon intercept. 


\section{Conclusion}

In summary, our relativistic many-body approach provides a glueball Regge trajectory similar to the pomeron diffraction fit. The model also unifies and describes the quark sector by properly implementing chiral symmetry (and breaking) to reproduce the observed meson spectra and attending Regge trajectories with the same, predetermined confining potential. Recent lattice measurements are also consistent with this picture and support a gluonic interpretation of the pomeron. Reproducing higher $J^{++}$states on the pomeron trajectory would further affirm this assertion and more sophisticated calculations are in progress along with a call for alternative model predictions and experimental measurements. Our results also indicate that the pomeron provides a worthwhile guiding constraint which future theoretical and experimental glueball studies should utilize. Finally, our approach offers an alternative explanation for the non-observance of the odderon in high energy nucleon scattering as well as for the absence of very light $C=-1$ glueballs.

\section{Acknowledgements}

Steve Cotanch thanks Frank Close for informative comments. Pedro Bicudo acknowledges enlightening pomeron discussions with Barbara Clerbaux, Brian Cox and Mike Pichowsky. This work is supported in part by grants DOE DE-FG02-97ER41048, DE-FG0287ER40365 and NSF INT-9807009. Felipe J. Llanes-Estrada was a SURA-JLab graduate fellowship recipient and thanks Katja Waidelich for technical help. Supercomputer time from NERSC is also acknowledged.

\section{References}

[1] I. Pomeranchuk, Sov. Phys. 3 (1956) 306;

L. Okun, I. Pomeranchuk, Sov. Phys. JETP 3 (1956) 307;

L. Foldy, R. Peirls, Phys. Rev. 130 (1963) 1585.

[2] I. Pomeranchuk, Sov. Phys. 7 (1958) 499.

[3] T. Regge, Nuovo Cimento 14 (1959) 951.

[4] P.D.B. Collins, An Introduction to Regge Theory and High Energy Physics, Cambridge Univ. Press, Cambridge, 1977.

[5] P.V. Landshoff, J.C. Polkinghorne, Nucl. Phys. B 32 (1971) 541;

A. Donnachie, P.V. Landshoff, Nucl. Phys. B 231 (1984) 189;

A. Donnachie, P.V. Landshoff, Nucl. Phys. B 244 (1984) 322;

A. Donnachie, P.V. Landshoff, Phys. Lett. B 185 (1987) 403;

A. Donnachie, P.V. Landshoff, Phys. Lett. B 437 (1998) 408.

[6] P.V. Landshoff, in: Proceedings of International Workshop on Deep Inelastic Scattering and Related Phenomena (DIS 96), DIS, 1996, p. 232.

[7] M.A. Pichowsky, T.-S.H. Lee, Phys. Lett. B 379 (1996) 1;

B. Clerbaux, Electroproduction Elastique de Mésons $\rho$ à HERA, PhD Thesis, 1999.

[8] C. Adolff, et al., Z. Phys. C 76 (1997) 613;

A. Donnachie, P.V. Landshoff, Phys. Lett. B 437 (1998) 408;

B.E. Cox, J.R. Forshaw, L. Lonnblad, hep-ph/0012310;

B. Clerbaux, private communication. 
[9] B.E. Cox, Nucl. Phys. (Proc. Suppl.) 79 (1999) 315.

[10] E. Kuraev, L. Lipatov, V. Fadin, Sov. Phys. JETP 45 (1977) 199;

Y. Balitskyand, L. Lipatov, Sov. J. Nucl. Phys. 28 (1978) 822.

[11] J. Forshaw, D. Ross, Quantum Chromodynamics and the Pomeron, Cambridge Univ. Press, Cambridge, 1997.

[12] A. Mueller, et al., Nucl. Phys. B 415 (1994) 373;

A. Mueller, et al., Nucl. Phys. B 425 (1994) 471;

N.N. Nikolaev, et al., Zeit. Phys. C 53 (1992) 331;

N.N. Nikolaev, et al., Phys. Lett. B 378 (1996) 347.

[13] G.F. Chew, S.C. Frautschi, Phys. Rev. Lett. 7 (1961) 394.

[14] L. Kisslinger, W. Ma, Phys. Lett. B 485 (2000) 367.

[15] A. Kaidalov, Y. Simonov, Phys. Lett. B 477 (2000) 163.

[16] L.D. Soloviev, Theor. Math. Phys. 126 (2001) 203.

[17] R.C. Brower, S.D. Mathur, C. Tan, hep-ph/0003153.

[18] M.M. Brisudova, L. Burakovsky, T. Goldman, Phys. Rev. D 58 (1998) 114015.

[19] S. Abatzis, et al., Phys. Lett. B 324 (1994) 509.

[20] WA 102 Collaboration, D. Barberis, et al., Phys. Lett. B 474 (2000) 423;

A. Kirk, Phys. Lett. B 489 (2000) 29.

[21] I. Bars, A.J. Hanson, Phys. Rev. D 13 (1974) 1744;

J. Johnson, C.B. Thorn, Phys. Rev. D 13 (1976) 1934.

[22] F. Iachello, N.C. Mukhopadhyay, L. Zhang, Phys. Rev. D 44 (1991) 898.

[23] A.P. Szczepaniak, E.S. Swanson, C.-R. Ji, S.R. Cotanch, Phys. Rev. Lett. 76 (1996) 2011.

[24] F.J. Llanes-Estrada, S.R. Cotanch, Phys. Rev. Lett. 84 (2000) 1102.

[25] F.J. Llanes-Estrada, S.R. Cotanch, Nucl. Phys. A 697 (2001) 303.

[26] F.J. Llanes-Estrada, S.R. Cotanch, Phys. Lett. B 504 (2001) 15.

[27] E. Gubankova, C.-R. Ji, S.R. Cotanch, Phys. Rev. D 62 (2000) 074001.

[28] C.J. Morningstar, M. Peardon, Phys. Rev. D 60 (1999) 034509.

[29] UKQCD Collaboration, G.S. Bali, et al., Phys. Lett. B 309 (1993) 378.

[30] H. Chen, J. Sexton, A. Vaccarino, D. Weingarten, Nucl. Phys. B 34 (1994) 357.

[31] M. Teper, hep-th/9812187.

[32] WA 102 Collaboration, D. Barberis, et al., Phys. Lett. B 467 (1999) 165.

[33] F.E. Close, G.A. Schuler, Phys. Lett. B 464 (1999) 279.

[34] D.Q. Liu, J.M. Wu, hep-lat/0105019.

[35] L. Lukaszuk, B. Nicolescu, Nuovo Cimento Lett. 8 (1973) 405.

[36] J.R. Cudell, et al., Phys. Rev. D 61 (2000) 034019.

[37] M. Reuter, H.G. Dosch, Phys. Lett. B 380 (1996) 177.

[38] E.R. Berger, A. Donnachie, H.G. Dosch, W. Kilian, O. Nachtmann, M. Rueter, Eur. Phys. J. C 9 (1999) 491. [39] E.R. Berger, A. Donnachie, H.G. Dosch, O. Nachtmann, Eur. Phys. J. C 14 (2000) 673. 